\title{
Culminación de la colaboración transfronteriza: Geoportal OTALEX C.
}

\author{
Marcos Soriano* \\ Carmen Caballero** \\ Rafael Álvarez ${ }^{* *}$ \\ Teresa Batista** \\ Júlio Mateus*** \\ Pedro Vivas ${ }^{* * *}$
}

Recibido el 14 de marzo de 2017; aceptado el 22 de mayo de 2017

\begin{abstract}
After many years of joint work, the regions of Extremadura (Spain), Alentejo (Portugal) and later Centro (Portugal), in search of a few principles as inspiring as the relevance of environmental value and the economic and social development, show the peculiarities of the territory through the OTALEX C Geoportal. In this there are publications made in the different joint projects and cartographic and thematic information displayed from different data viewers, which put in hands of the user the necessary tools to discover and interpret correctly the territory.

Key words: Cartography, SDI, Geoportal, Spatial Planning, Cross-border Areas.

\section{Resumo}

Depois de muitos anos de trabalho conjunto, as regiões da Extremadura (Espanha), Alentejo (Portugal) e posteriormente Centro (Portugal), em busca de princípios tão inspiradores como da relevância do valor ambiental e o desenvolvimento econômi-

* GISVESA, S.A., correo electrónico: marcos.soriano@gisvesa.com

** Centro de Información Cartográfica y Territorial de Extremadura, Consejería de Medio Ambiente y Rural, Políticas Agrarias y Territorio, Junta de Extremadura, correo electrónico: carmen.caballeroc@juntaex.es, ralvarez@juntaex.es

*** Comunidade Intermunicipal do Alentejo Central, correos electrónicos: tbatista@cimac.pt, jmateus@cimac.pt

**** Instituto Geográfico Nacional de España, Centro Nacional de Información Geográfica, correo electrónico: pedro.vivas@cnig.es
\end{abstract}


co e social, mostram as particularidades do território através do Geoportal OTALEX C. Nele se citam desde as publicações realizadas nos diferentes projetos conjuntos até a informação cartográfica e temática que se mostra a partir de diferentes visualizadores de dados, pondo nas mãos dos usuários as ferramentas necessárias para descobrir e interpretar de forma correta o território.

Palavras chave: Cartografia, IDE, Geoportal, Ordenação do Território, Espaços Fronteiriços.

\section{Resumen}

Tras muchos años de trabajo conjunto, las regiones de Extremadura (España), Alentejo (Portugal) y posteriormente Centro (Portugal), en busca de unos principios tan inspiradores como la relevancia del valor medioambiental y el desarrollo económico y social, muestran las particularidades del territorio a través del Geoportal OTALEX C. En él se dan cita desde las publicaciones realizadas en los diferentes proyectos conjuntos hasta la información cartográfica y temática que se muestra a partir de diferentes visualizadores de datos, poniendo en manos del usuario las herramientas necesarias para descubrir e interpretar de forma correcta el territorio.

Palabras clave: Cartografía, IDE, Geoportal, Ordenación del Territorio, Espacios Fronterizos.

\section{Evolución de la colaboración transfronteriza}

En el año 1997 mediante el proyecto "Coordinación de Sistemas de Información Geográfica y de los Instrumentos de Observación Territorial en Espacios Transfronterizos de Baja Densidad (COORDSIG)” comienza la cooperación transfronteriza en materia de Territorio y Sistemas de Información Geográfica entre las regiones de Alentejo en Portugal y Extremadura en España, sentando las bases de futuros proyectos no solo mediante la puesta en común de una cartografía básica única a ambos lados de la frontera si no por la formación de un equipo multidisciplinar que involucra a los tres niveles de la administración (nacional, regional y local). Posteriormente proyectos como PLANEXAL (2003-2005), en el que se estudian las parcelaciones, urbanizaciones y edificaciones a los perímetros urbanos o GEOALEX (2004-2006), que persigue la obtención de un modelo geográfico de gestión ambiental y territorial para espacios rurales de baja densidad, abundan en el conocimiento del territorio común potenciando las fortalezas transfronterizas.

El proyecto OTALEX (2006-2009) plantea un Observatorio Territorial en el que por un lado se establece el estudio de la sostenibilidad a partir de una serie de indicadores y por otro la creación de una Infraestructura de Datos Espaciales (IDE) en la que poner a disposición del usuario los datos tanto geográficos como temáticos obtenidos siguiendo las orientaciones de la Directiva INSPIRE. Dicho Geoportal 
presenta los tres módulos básicos de una IDE: visualizador de datos geográficos, búsqueda de nombres geográficos y Catálogo de metadatos.

Durante el proyecto OTALEX II (2008-2011) se produce la consolidación del Observatorio Territorial y el enriquecimiento del Geoportal dotándolo de información ambiental, así como herramientas para la gestión avanzada de usuario, monitorización ambiental, geoprocesamientos mediante el estándar WPS y carga de datos externos entre otros.

La madurez del proyecto culmina durante el transcurso del proyecto OTALEX C (2010-2013) en el que la ampliación del ámbito territorial sumando la región Centro de Portugal supone una apuesta por la consolidación de la EUROACE. En cuanto al Geoportal, continúa su evolución añadiendo un nuevo visualizador de datos centrado en el estudio de los indicadores, renueva su apuesta por el I+D mediante el estudio de modelos ontológicos para Datos OTALEXC al objeto de su publicación en la web semántica LinkedData así como la publicación en la IDEOTALEX de mapas temáticos en tiempo real con datos medioambientales capturados por sensores en campo recibidos a través del estándar SOS 2.0.

A continuación, veremos con más detalle cada una de las partes del Geoportal OTALEX C.

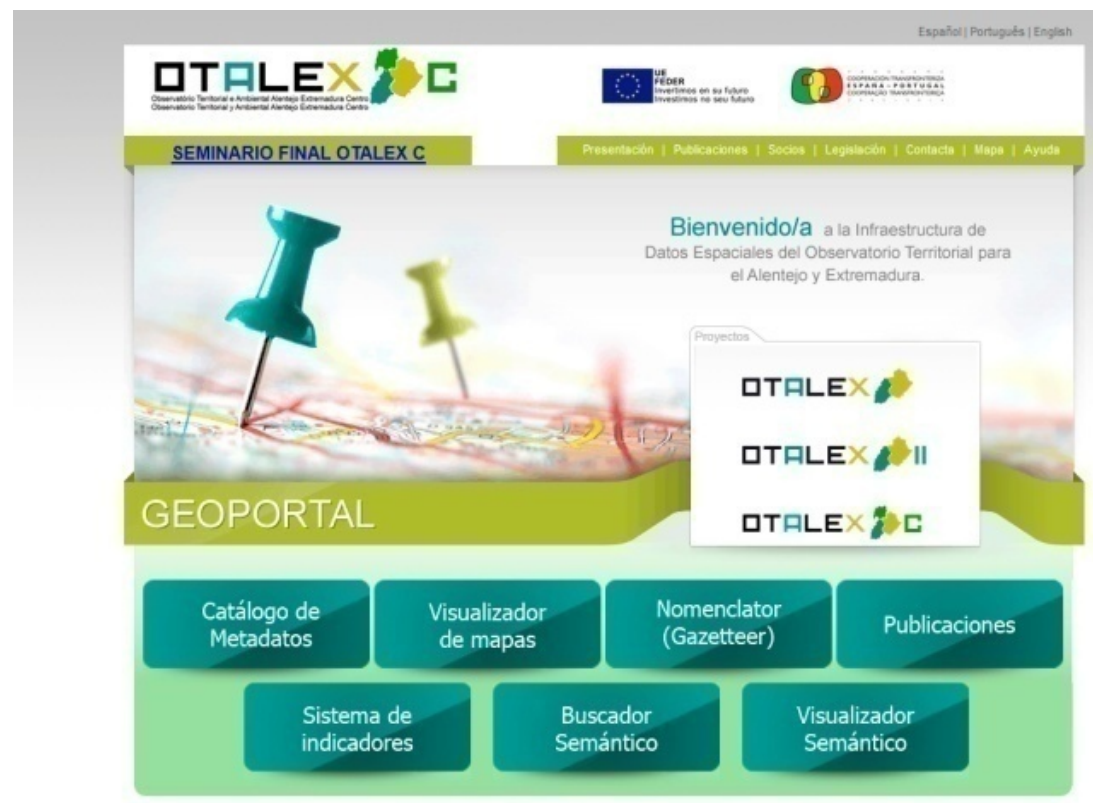

Figura 1. Vista del Geoportal OTALEX C, <http://www.ideotalex.eu/OtalexC/> . 


\section{Catálogo de Metadatos}

Permite descubrir la información contenida en la IDE a partir de sus metadatos, realizando búsquedas por palabras y aplicando filtros de categoría o geográficos.

\section{Visualizador de Mapas}

El Visualizador de Mapas, ha recorrido un camino paralelo a la consecución de los diferentes proyectos acometidos, enriqueciéndose con nuevos datos, mejorando en cada uno de ellos las funcionalidades implementadas en la versión anterior y aportando nuevas herramientas adaptándose a las necesidades de los usuarios.

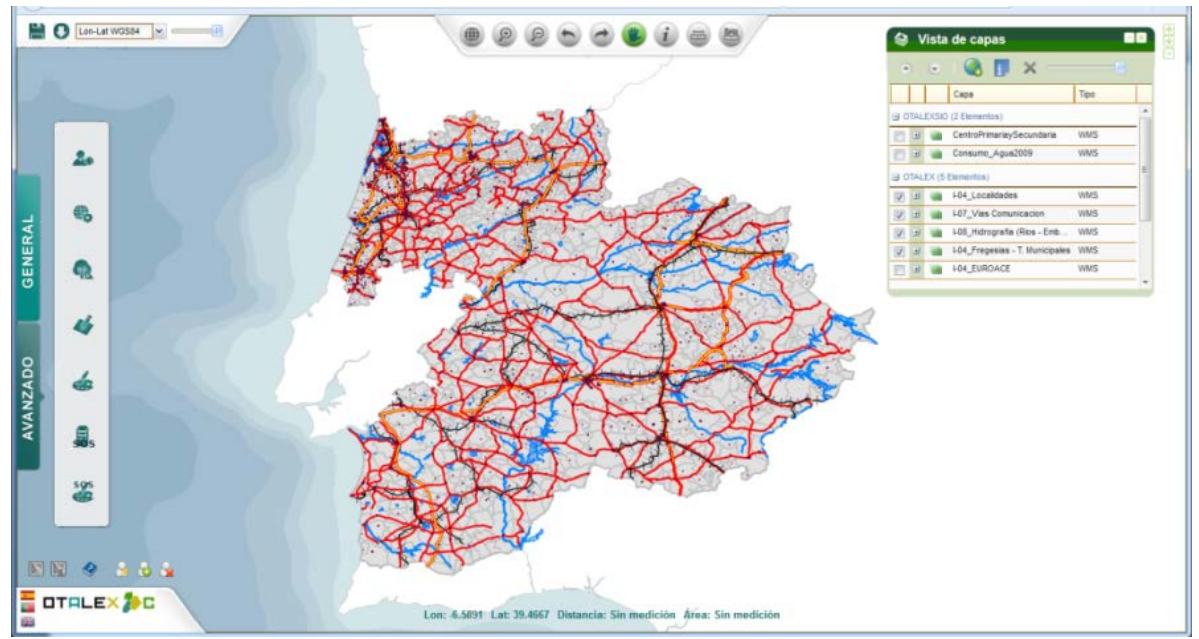

Figura 3. Vista general del Visualizador de Mapas, $<$ http://www.ideotalex.eu/OtalexC/PortalOtalex/Visor.html>

Con objeto de dar una mayor importancia a la información gráfica el acceso a las diferentes funcionalidades se ha repartido en el contorno del visualizador y a ser posible en ventanas desplegables, de esta manera podemos destacar tres bloques:

- Herramientas de navegación: centrada en la parte superior da acceso a las diferentes funciones de zoom, consulta de elementos y medición de áreas y distancias.

- Vista de capas: situada en la parte derecha, permite la activación de las capas del nodo central, así como añadir nuevas capas tanto de los servidores de los socios como cualquier otro servicio estándar (WMS).

- Funcionalidades: las pestañas situadas en la parte izquierda dan acceso a las herramientas agrupadas en "General” y “Avanzado”. Desde ellas podremos ac- 
tivar las capas, agregar nuevos servicios, generar mapas impresos, consultar las capas sociales creadas a partir de la aplicación IDE móvil, aplicar geoprocesos (WPS), dibujar geometrías (líneas, puntos y polígonos), crear consultas complejas sobre capas WFS o conectar con redes de sensores (SOS) entre otras.

\section{Nomenclátor}

Esta aplicación permite la consulta de la base de datos de nombres geográficos del proyecto a partir de palabras con la posibilidad de aplicar filtros alfanuméricos y geográficos.

\section{Publicaciones}

Da acceso al conjunto de publicaciones realizadas en los diferentes proyectos acometidos.

\section{Sistema de Indicadores de la IDE de OTALEX C (SIO)}

Uno de los objetivos prioritarios del proyecto desde sus inicios consiste en la caracterización y análisis del territorio, así como la monitorización de las alteraciones y presiones a ambos lados de la frontera hispano-portuguesa. Este trabajo se afianza en el estudio a partir de indicadores agrupados según cuatro ámbitos: Territorial, Ambiental, Social y Económico. La conjunción de estos cuatro ámbitos nos permite a su vez definir el territorio desde el punto de vista de la Sostenibilidad.

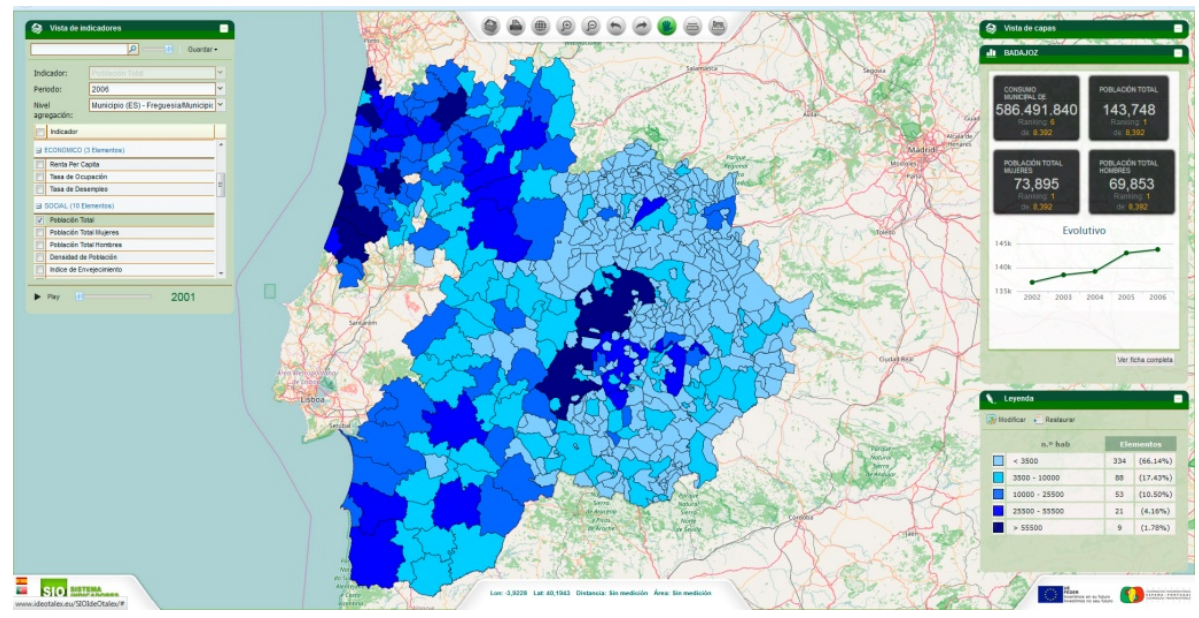

Figura 5. Ventana principal SIO, <http://www.ideotalex.eu/SIOIdeOtalex/>. 
Se define, por tanto, el SIO, como el conjunto de indicadores de la IDE OTALEX junto con las herramientas necesarias para su almacenamiento, gestión, explotación y difusión. Este sistema permite ofrecer un conjunto de gráficas estadísticas y mapas temáticos que, presentados sobre la cartografía, complementan las capacidades de la plataforma existente.

\section{Buscador semántico y Visualizador semántico}

En el transcurso del proyecto OTALEX C se realiza la generación, publicación y enlazado en Linked Data de los datos correspondientes a estadística, hidrografía e información territorial básica. El Buscador semántico consiste en un editor de consultas que permite explorar la web semántica. En cuanto al Visualizador semántico permite consultar la información geográfica almacenada en la web semántica.

\section{Portal de datos abiertos}

La siguiente regeneración que se está acometiendo es la transformación en portal de datos abiertos implantándose los estándares referentes a este tipo de servicios, reintegrando la infraestructura existente y la posibilidad de estrategias de federación para compartir los datos, tanto de cada uno de los socios, como del propio proyecto.

\section{Bibliografía}

Directiva 2007/2/CE del Parlamento Europeo y del Consejo, del 14 de marzo de 2007, por la que se establece una infraestructura de información espacial en la Comunidad Europea (INSPIRE), <http://www.europarl.europa.eu>.

Álvarez, R. Caballero; C., Ceballos; F. Soriano, M. (2009). "Una IDE transfronteriza y Observatorio Ambiental”, Revista Internacional de Ciencias de la Tierra MAPPING, no. 141, 2010, pp. 14-19.

Soriano, M. Caballero y C. Ramos, R. (2011). "Infraestructura de Datos Espaciales OTALEX. Camino de la madurez”, OTALEX II resultado final proyecto, pp. 17-28. DL: BA-000365-2011.

Caballero, C. Álvarez; R. Soriano, M.; Mateus, J. Vivas, P. Hernández, F.J. Reis, S. Roque, N. Lozano, D. (2013). "IDE OTALEX Arrojo, Constancia-Evolución, Innovación”, OTALEX C, resultados del proyecto, pp. 203-214. 